This item was submitted to Loughborough's Research Repository by the author.

Items in Figshare are protected by copyright, with all rights reserved, unless otherwise indicated.

\title{
Estimation of bluff body transient aerodynamics using an oscillating model rig
}

PLEASE CITE THE PUBLISHED VERSION

http://dx.doi.org/10.1016/j.jweia.2007.06.043

PUBLISHER

(c) Elsevier

VERSION

AM (Accepted Manuscript)

LICENCE

CC BY-NC-ND 4.0

REPOSITORY RECORD

Mansor, Shuhaimi, and Martin A. Passmore. 2010. "Estimation of Bluff Body Transient Aerodynamics Using an Oscillating Model Rig". figshare. https://hdl.handle.net/2134/6645. 
This item was submitted to Loughborough's Institutional Repository (https://dspace.lboro.ac.uk/) by the author and is made available under the following Creative Commons Licence conditions.

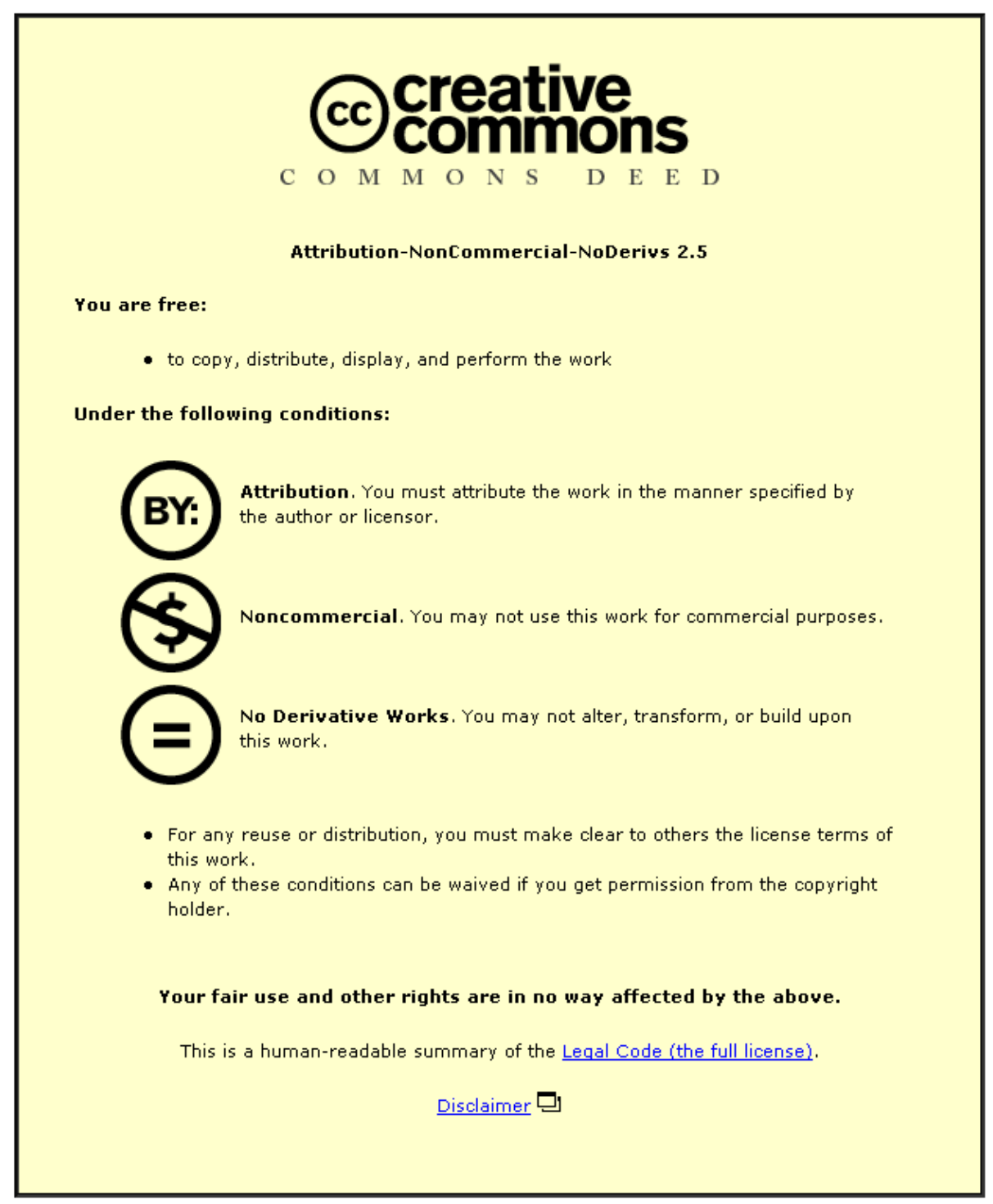

For the full text of this licence, please go to: http://creativecommons.org/licenses/by-nc-nd/2.5/ 


\title{
Estimation of Bluff Body Transient Aerodynamics Using an Oscillating Model Rig
}

\author{
S. Mansor, Researcher, M.A. Passmore, Senior lecturer \\ Department of Aeronautical and Automotive Engineering, Loughborough University, LE11 3TU, UK.
}

\begin{abstract}
A method for the estimation of transient aerodynamic derivatives from dynamic wind tunnel tests using time response data is presented in this paper. For the purposes of the study the aerodynamic derivatives are considered to act as a stiffness and damping to the model motion. The experimental set-up consists of a simple bluff body (Davis model) constrained to oscillate with a single degree of freedom of pure yawing motion. A range of springs were used to control the oscillation frequency and hence the reduced frequency. The transient responses from dynamic wind tunnel tests are compared with quasi-steady analysis in order to investigate the effect of unsteady aerodynamics. The aerodynamic derivatives are initially estimated using the classical logarithmic decay method. The dynamic stiffness derivative exceeds that determined statically across the reduced frequency range. The damping derivative was found to be a function of free-stream speed; at low velocities it is negative but progressively increases to a positive value. With further increases in speed, a selfsustained oscillation is observed with almost constant frequency and amplitude. This result is attributed to coupling between the model wake and the model stability; however, the exact mechanism of the interaction is not fully understood. This phenomenon is under further investigation.
\end{abstract}

Keywords: Transient aerodynamics, bluff body, aerodynamic derivatives, vortex shedding, reduced frequency, natural frequency, damping ratio.

\section{Nomenclature}

A - model frontal area

$C_{a}$ - aerodynamic damping

$C n$ - yaw moment coefficient

$C_{r}$ - mechanical damping

$\mathrm{Cn}_{\beta}$ - yaw moment derivative

$\mathrm{Cn}_{r}$ - yaw damping derivative

$f \quad$ - oscillation frequency

$I_{z z}$ - yaw inertia

$K_{a}$ - aerodynamic stiffness

$K_{m}$ - reduced frequency

$K_{r}$ - mechanical stiffness

$\ell \quad$ - model characteristic length

$m$ - model mass

$N_{\beta}$ - dimensional yaw moment

$\hat{N}_{\beta}$ - dynamic normalised yaw moment

$\hat{N}_{r}$ - dynamic normalised yaw damping

$r$ - yaw rate

$t$ - time

$T$ - period of oscillation

$V \quad$ - wind tunnel speed

$\beta$ - model yaw angle

$\zeta$ - damping ratio

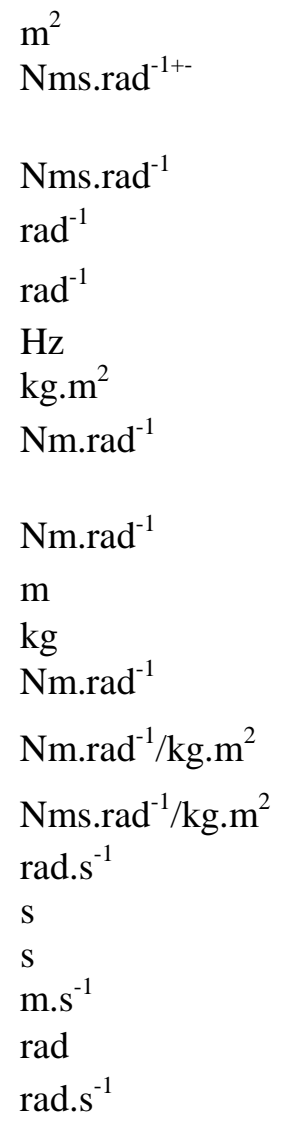

$\mathrm{m}^{2}$

Nms.rad

$\mathrm{rad}^{-1}$

kg.m ${ }^{2}$

Nm.rad ${ }^{-1}$

$\mathrm{kg}$

$\mathrm{Nms}^{-1} / \mathrm{kg} \mathrm{m}^{2}$

rad.s ${ }^{-1}$

$\mathrm{s}$

$\operatorname{rad} . \mathrm{s}^{-1}$ 
$\rho \quad$ - air density

$\omega$ - oscillation frequency

$\omega_{d}$ - damped frequency

$\omega_{n}$ - natural frequency kg. $\mathrm{m}^{-3}$

rad.s $s^{-1}$

rad.s $s^{-1}$

rad.s ${ }^{-1}$

\subsection{Introduction}

The transient aerodynamic forces and moments acting on a road vehicle have been the subject of interest for many years because of the implications for vehicle safety and refinement. However, the transient aerodynamics are not well understood with some studies reporting that the steady state loads are a conservative estimate of the dynamic case (Bearman and Mullarkey, 1994; Garry and Cooper, 1986), while others indicate overshoots under transient conditions, particularly in the yaw moment response (Chadwick et al, 2000; Passmore et al' 2001; Russell, 1969; Ryan and Dominy 1998). Thus, in this paper the dynamic response of a simple automotive bluff body, measured using an oscillating model rig, is compared with the response of the same model measured using static tests. In both cases the response is characterised by calculating the aerodynamic derivatives from the test. A schematic of the experimental setup is shown in Fig.1, it consists of a simple bluff body

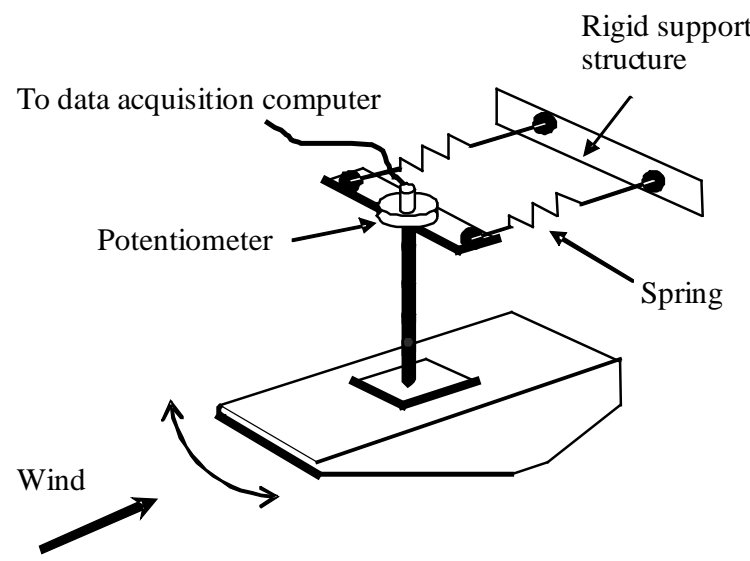

constrained to oscillate with a single degree of freedom of pure yawing motion. The rig is mounted to the tunnel working section roof.

Figure 1. Oscillating model rig.

The paper describes the initial development of the method for estimating the transient aerodynamic derivatives from time response data acquired during the dynamic wind tunnel tests. For the purposes of this analysis the aerodynamic derivatives are considered to act as a stiffness and damping to the model motion.

The frequency range of importance in crosswind studies has been identified as approximately 0.2 to $2.0 \mathrm{~Hz}$ (Goetz, 1995) at full scale. At motorway speeds this corresponds to reduced frequencies between approximately 0.03 and 0.3 where reduced frequency is defined as:

$$
K_{m}=\frac{f L}{V}
$$

To ensure similitude of the periodic flows these reduced frequencies are matched in the experimental setup by altering the stiffness of the springs shown in the apparatus. 
The effect of Reynolds number on the derivatives is also investigated by varying the tunnel free stream velocity.

\section{Experimental set up.}

The wind tunnel tests were undertaken in the $1.92 \times 1.32 \mathrm{~m}$, closed working section, low speed, open-circuit wind tunnel in the Department of Aeronautical and Automotive engineering at Loughborough University. The oscillator mechanism is mounted to a rigid support structure outside the working section and the circular section steel rod, of $20 \mathrm{~mm}$ diameter, passes through a clearance hole in the ceiling. The model is mounted to the end of the support rod and is free to rotate in yaw. The combination of the tunnel flow and the model oscillation then provides an unsteady wind input.

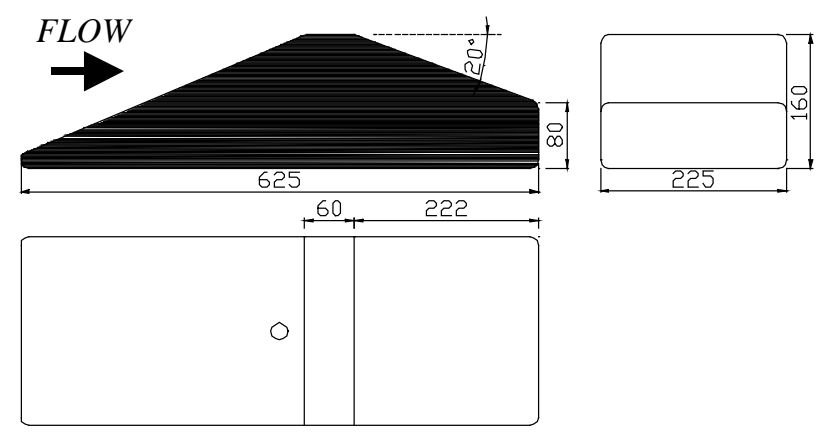

Figure 2. Davis model geometry

The model employed in the study is a simplified road vehicle shape, (Davis model) constructed from fibreglass. This model was previously used by Passmore et al, 2001, to estimate aerodynamic derivatives from unsteady surface pressure arising from a sinusoidal gust input. The model dimensions are shown in Fig.2. In this application the model geometric blockage is approximately $1.4 \%$.

During set up an adjustment mechanism, on the two linear springs attached to the cross arm, allows the spring forces to be equalised and the model to be aligned in the tunnel. The angular position of the model is recorded using a low friction potentiometer mounted to the top of the support rod. The mass $(m)$ and moment of inertia $\left(I_{z z}\right)$ of the model are $4.215 \mathrm{~kg}$ and $0.102 \mathrm{kgm}^{2}$ respectively. The Reynolds number range is between $0.42 \times 10^{6}$ to $1.68 \times 10^{6}$ based on model length. The test can be conducted using two longitudinal positions of the axis of rotation in order to make possible the estimation of the side force derivatives, however, this paper is restricted to the study of the yaw moment derivatives.

\subsection{Static test}

The static yaw aerodynamic derivatives were obtained from a conventional yaw test with the model mounted on a 6-component balance. The derivative, in this case the gradient of the yaw moment versus yaw angle response, were calculated over a yaw angle range consistent with that generated in the dynamic tests. Fig. 3 shows the result of the static tests and Table 1 shows the gradient (yaw moment derivative) obtained from each test. 


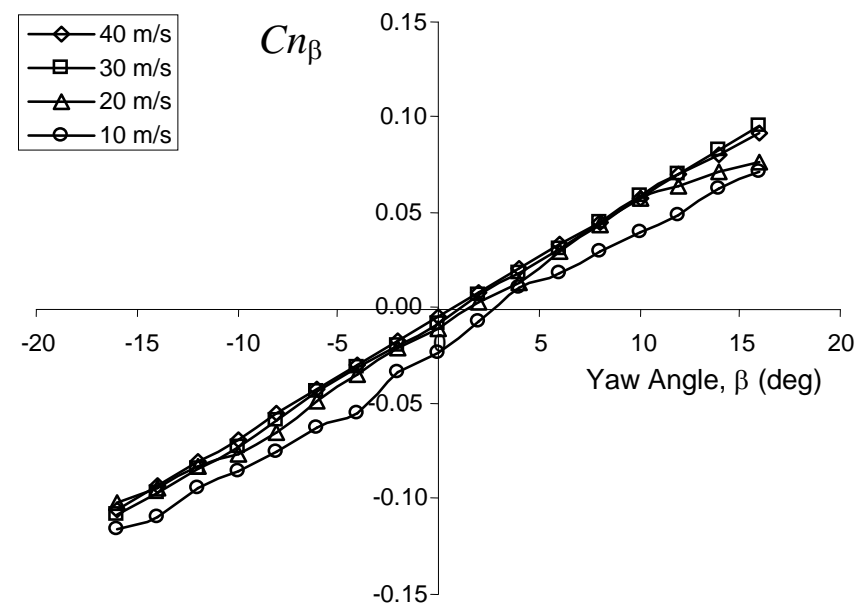

Figure 3 Yaw moment coefficient against yaw angle

\begin{tabular}{|c|c|}
\hline Wind Speed $(\mathrm{m} / \mathrm{s})$ & $C_{n_{\beta}}\left(\mathrm{rad}^{-1}\right)$ \\
\hline 10 & 0.3782 \\
\hline 20 & 0.3839 \\
\hline 30 & 0.3667 \\
\hline 40 & 0.3610 \\
\hline \multicolumn{2}{|c|}{ Table 1 } \\
\hline
\end{tabular}

\subsection{Dynamic test}

In the dynamic tests a free oscillation is initiated by giving the model an initial yaw angle $\beta_{0}$, and releasing it. The time history of yaw angle is recorded and the test is repeated for a wind-on and a wind-off condition. In the case of the wind-on condition the motion is determined by the combination of the effect of the unsteady aerodynamic loads and any mechanical terms that arise from the rig. If it is assumed that the mechanical terms can be determined from the wind-off experiment and that the aerodynamic terms can be considered only as aerodynamic stiffness and damping terms the motion can be analysed. Let $K_{a}$ and $C_{a}$ represent the aerodynamic stiffness and damping respectively;

$$
\begin{aligned}
& K_{a}=\frac{1}{2} \rho V^{2} A \ell C n_{\beta} \\
& C_{a}=\frac{1}{2} \rho V^{2} A \ell C n_{r} \frac{\ell}{V}
\end{aligned}
$$

For the purpose of derivation, we assume that the aerodynamic stiffness and damping are positive. The single degree-of-freedom equation of motion is then written as,

$$
I_{z z} \ddot{\beta}+C_{r} \dot{\beta}+K_{r} \beta=K_{a} \beta+C_{a} r
$$

substituting $r=\dot{\beta}$ in Eq.(4) and rearranging, yields, 


$$
\ddot{\beta}+\frac{C_{r}-C_{a}}{I_{z z}} \dot{\beta}+\frac{K_{r}-K_{a}}{I_{z z}} \beta=0
$$

\section{Estimation of the aerodynamic derivatives}

From the time responses the aerodynamic damping and stiffness can be calculated by substituting the period of the oscillation and time to half amplitude of the wind-off and windon in Eq.(6) and (7). The wind-off measurement accounts for the non-aerodynamic terms.

$$
\begin{aligned}
& \hat{N}_{\beta}=-\left\{4 \pi^{2}\left(\frac{1}{T_{\text {on }}{ }^{2}}-\frac{1}{T_{\text {off }}^{2}}\right)+0.6931^{2}\left[\frac{1}{\left(t_{1 / 2}\right)_{\text {on }}^{2}}-\frac{1}{\left(t_{1 / 2}\right)_{\text {off }}^{2}}\right]\right\} \\
& \hat{N}_{r}=-1.3863\left[\frac{1}{\left(t_{1 / 2}\right)_{\text {on }}}-\frac{1}{\left(t_{1 / 2}\right)_{\text {off }}}\right]
\end{aligned}
$$

where the subscript off and on represent wind-off and wind-on conditions respectively. Eq.(6) can be modified by substituting the period of oscillation in the form of oscillation frequency, rearranging in terms of frequency ratio between wind-on and wind-off. The nondimensional aerodynamic derivatives of $\mathrm{Cn}_{\beta}$ and $\mathrm{Cn}_{r}$ are then calculated using the following expressions [5],

$$
C n_{\beta}=\frac{\hat{N}_{\beta} I_{z z}}{\frac{1}{2} \rho V^{2} A \ell} \quad \text { (8) } \quad C n_{r}=\frac{\hat{N}_{r} I_{z z} V}{\frac{1}{2} \rho V^{2} A \ell^{2}}
$$

As the estimation of the aerodynamic derivatives is referenced to the body axis of the model, then the aerodynamics derivatives $C n_{\beta}$ and $C n_{r}$ are directly comparable with those determined in the static test.

\section{Quasi steady analysis}

The quasi-steady response of the model is defined as the dynamic response predicted using the steady state yaw-moment data. From the equation of motion;

$$
\sum \text { Yaw Moment }=I_{z z} \ddot{\beta}
$$

the transfer function can be derived by substituting appropriate initial conditions. If the initial yaw angle is $\beta_{0}$ and the model is released from rest then $\beta(0)=\beta_{0}$ and $\dot{\beta}(0)=0$. Expanding Eq.(5) in its Laplace form, yields,

$$
\beta(s)=\frac{\beta_{o}\left[s+\frac{\left(C_{r}-C_{a}\right)}{I_{z z}}\right]}{\left[s^{2}+\frac{\left(C_{r}-C_{a}\right)}{I_{z z}} s+\frac{\left(K_{r}-K_{a}\right)}{I_{z z}}\right]}
$$

Eq.(11) takes the form of a simple second-order dynamic system, so by inspection the natural frequency and damping ratio can be written as:

$$
\text { Natural Frequency, } \omega_{n}=\sqrt{\frac{\left(K_{r}-K_{a}\right)}{I_{z z}}}
$$




$$
\text { Damping Ratio, } \quad \zeta=\frac{C_{r}-C_{a}}{2 I_{z z} \sqrt{\frac{\left(K_{r}-K_{a}\right)}{I_{z z}}}}
$$

The mechanical torsional stiffness $K_{r}$ is determined from the linear spring stiffness and the cross arm length. The moment of inertia of the model system was determined experimentally from the gradient of the graph shown in Fig. 4 from two sets of tests T1 and T2. The moment of inertia about the axis of rotation is $0.102 \mathrm{kgm}^{2}$.

Figure 4. Torsional stiffness against natural frequency. Wind-off test.

\section{Results}

Results have been obtained for the system over the range of reduced frequencies indicated by the literature. Table 2 shows the range of springs used throughout the experiment and the natural frequency each imparts to the basic system.

\begin{tabular}{|l|l|l|l|l|l|l|l|l|l|l|}
\hline Spring & K1 & K2 & K3 & K4 & K5 & K6 & K7 & K8 & K9 & K10 \\
\hline Ks (N/m) & 49 & 119 & 214 & 306 & 806 & 1051 & 1751 & 2242 & 2594 & 3399 \\
\hline$f_{n_{\text {off }}}(\mathrm{Hz})$ & 0.51 & 0.79 & 1.06 & 1.27 & 2.07 & 2.37 & 3.06 & 3.46 & 3.72 & 4.26 \\
\hline
\end{tabular}

Table 2 Spring data

Example time response data is shown for three springs in Fig.5. The effect of the aerodynamic damping is seen clearly in the data. 

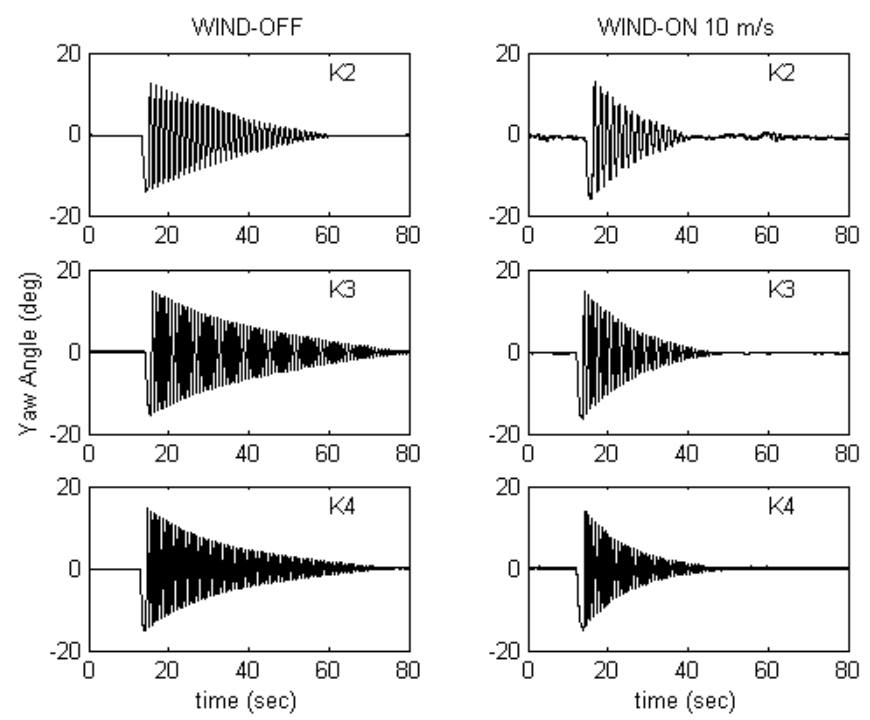

Figure 5 Example damped time response data.

Fig. 6 compares the wind-off and wind-on damping ratio at $10 \mathrm{~m} / \mathrm{s}$ tunnel speed for all ten springs. For wind-off it is seen to be very small indicating that the mechanical damping is small, this ensures that the system is sensitive to any aerodynamic damping that may be present in the wind-on tests. In the wind-on condition the aerodynamic contribution to the damping is seen to be significant at low oscillation frequencies, but at higher frequencies the damping is small in both cases. However, the presence of some aerodynamic damping can be seen across the frequency range tested.

Figure 6 Comparison of wind-on and wind-off damping ratios.

The frequency ratio ( $\omega_{\text {on }} / \omega_{\text {off }}$ ) between wind-on and wind-off is shown for all ten springs at a speed of $10 \mathrm{~m} / \mathrm{s}$ in Fig.7. These are compared to the frequency ratio predicted from a quasi-steady analysis with the aerodynamic derivative $\mathrm{Cn}_{\beta}$ taken from the steady state data at $10 \mathrm{~m} / \mathrm{s}$ and zero aerodynamic damping. The difference at low reduced frequency arises because of the difference between the static and dynamic aerodynamic derivative. It is also evident that the transient aerodynamic derivative is a function of reduced frequency. For reduced frequencies greater than about 0.1 the frequency ratio approaches unity and the quasi-steady and experimental frequency ratio are in agreement. 
Figure 7. Frequency ratio at $10 \mathrm{~m} / \mathrm{s}$.

The results in the previous figure were all obtained at a tunnel speed of $10 \mathrm{~m} / \mathrm{s}$. However at higher tunnel speeds the damped response was not always seen. Fig.8 shows the response for a single spring, K5, over a range of tunnel speeds. It is evident that above a critical speed the oscillation ceases to be damped and a self-sustained oscillation occurs. At higher speeds still, $(40 \mathrm{~m} / \mathrm{s})$ no initial displacement of the model is required to generate the oscillation, this is referred to as a self-excited oscillation. The transition away from the damped response is independent of reduced frequency but rather depends on the tunnel speed or Reynolds number.
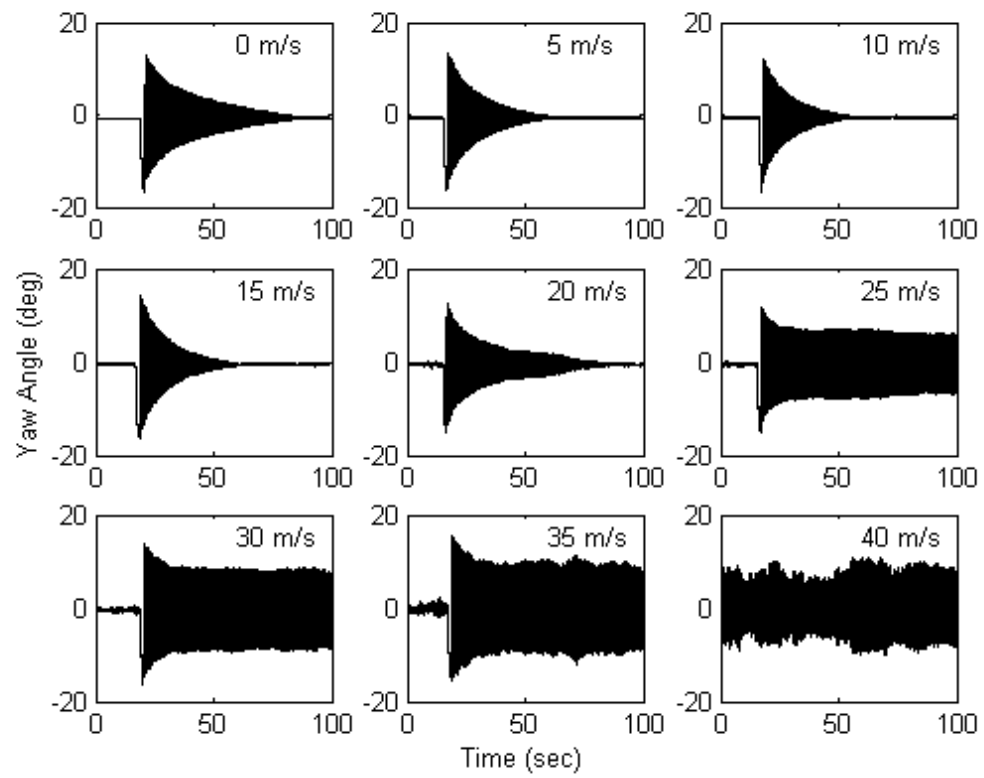

Figure 8.Time response for a single spring over a range of tunnel speeds.

In order to analyse these responses Eq.(6) is modified by substituting in oscillation frequency for period. By ignoring the damping term the aerodynamic stiffness can be written as: 


$$
N_{\beta}=-4 \pi^{2} f_{\text {off }}^{2}\left[\left(\frac{f_{\text {on }}}{f_{\text {off }}}\right)^{2}-1\right]
$$

For each un-damped case the wind-on oscillation frequency is obtained by generating the power spectral density from the time response.

Figure 9. showing collapse of data onto single curve

Fig.9 shows the frequency ratio against reduced frequency as in Fig.7 but in this case only for springs K5, to K10 and at a number of different wind speeds. The trends are the same as seen previously and the figure confirms that using reduced frequency collapses the data onto a single curve very effectively. Fig.10 summaries all the yaw moment aerodynamic derivative results. The data is presented in the form of a magnification factor, $C n_{\beta \text { dynamic }} / C n_{\beta \text { static }}$, as in Passmore et al, 2001.

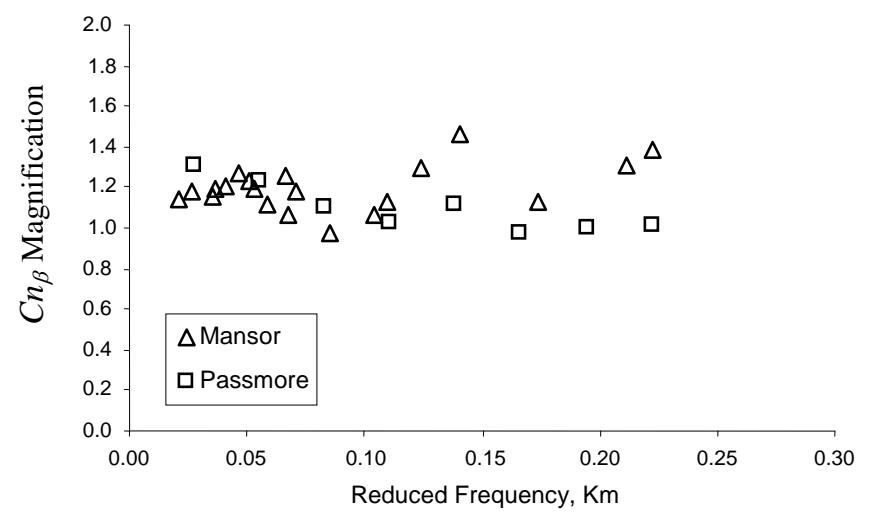

Figure 10 Yaw moment derivative magnification.

The magnification is greater than unity across the reduced frequency range in some instances by as much as 50\%. The data of Passmore et al, 2001, is included on the plot and shows good agreement below reduced frequencies of about 0.1. Above this the data of Passmore shows somewhat lower values, this is the region where, in the experiments reported here, the frequency ratio tends to unity. It should be noted that these results are produced from two quite different transient simulations and that although the tests are on the 
same model, they have been obtained in different wind tunnels with different flow quality, blockage ratios and over different yaw angle ranges. It is also noted that in some cases the gust amplitude used in the test by Passmore et al. [7] is less than $\pm 2^{\circ}$, which may affect the overall signal-to-noise ratio.

The yaw damping derivative $C n_{r}$ has been extracted from those time series that show a damped response and the results are shown in Fig.11.

Figure 11 Yaw damping derivative against reduced frequency.

The aerodynamic damping derivative is generally small apart from at the lowest speed and reduced frequency. At a speed between 15 and $20 \mathrm{~m} / \mathrm{s}$ the sign of the aerodynamic damping derivative is seen to become positive. It is suggested that the reduction in damping derivative with increasing speed arises as the strength of the vortex shedding increases. The positive damping value physically arises because the vortex shedding essentially drives the oscillation. However, whilst this effect is relatively small the overall response remains damped because of the influence of the mechanical damping, this also makes it possible to extract the value of aerodynamic damping from the time series.

The yaw moment derivative is plotted again in Fig.12 but in this case against Reynolds number. For all oscillation frequencies (springs) the yaw moment derivative reduces as Reynolds number increases and becomes almost constant above about $1 \times 10^{6}$.

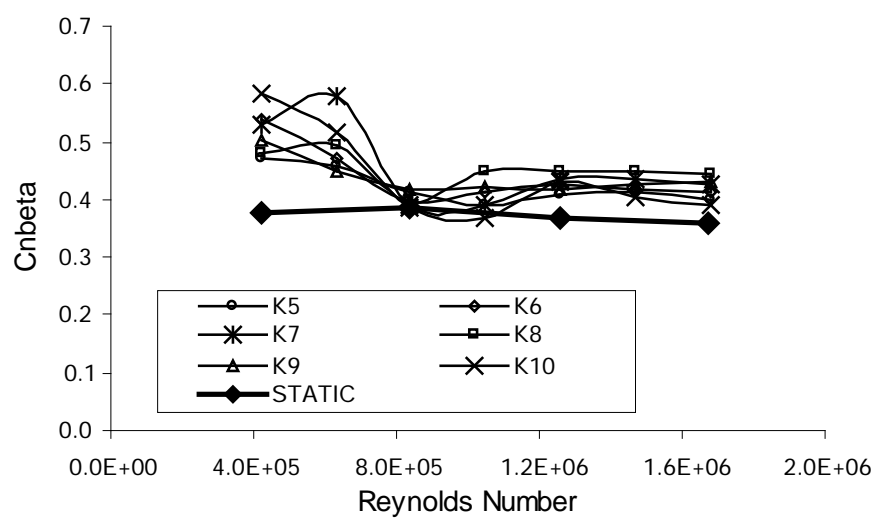

Figure 12. Yaw moment derivative against Reynolds number. 
Developing a model that accurately reflects the measured response is a priority in the future work. Fig. 13 shows the time and frequency responses of the model oscillation for $\mathrm{K} 5$ at $10 \mathrm{~m} / \mathrm{s}$. The simulation takes the basic quasi steady simulation but uses the measured dynamic moment and damping derivatives.
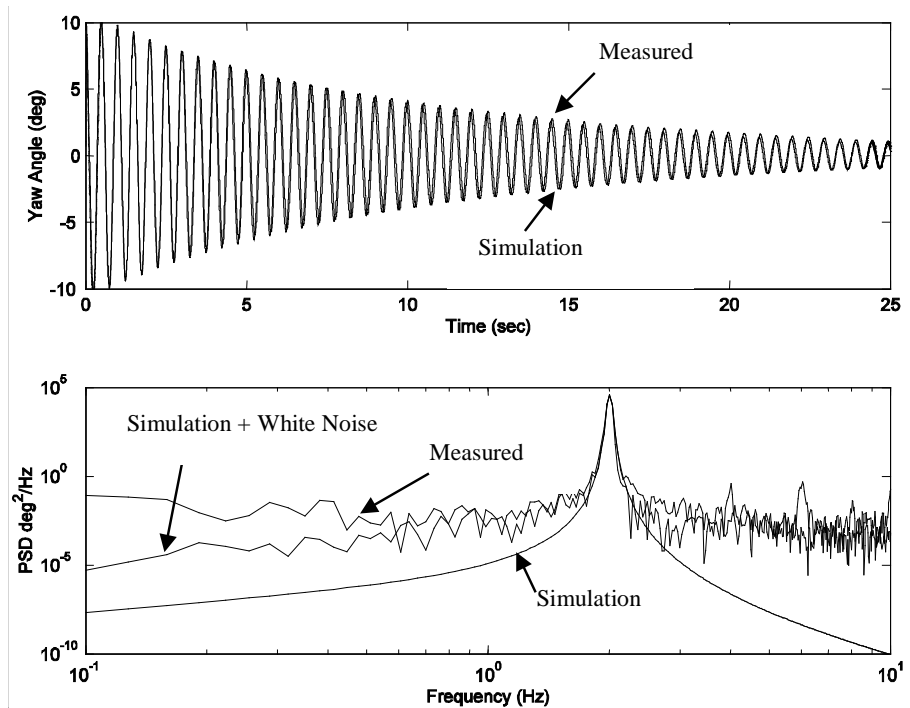

Figure 13. Comparison between simulation and measured results.

The simulation matches the fundamental frequency well, and the addition of white noise suggests that some of the discrepancy can be explained through the presence of noise in the measured data. However, it is also clear that even in this case, which exhibits a well behaved damped response, a significant unsteady component exists that requires further analysis.

Using the same simulation it can be shown that the self sustained oscillation occurs when the aerodynamic damping cancels out the mechanical damping. This is consistent with the results seen in Fig. 11 and 12 and shows that the speed at which self-sustained oscillation occurs is a function of the rig design and not a significant aerodynamic effect.

Fig. 14 compares the simulated response with the measured during a self sustaining oscillation. Clearly the simple simulation used here does not predict the unsteadiness seen in the self sustaining responses, but it is also evident that at higher speeds the unsteadiness is increasing. This is seen in the RMS yaw angle data. The conclusion that might be drawn from Fig.12 is that the transient behaviour is constant above a Reynolds number of $10^{6}$. 

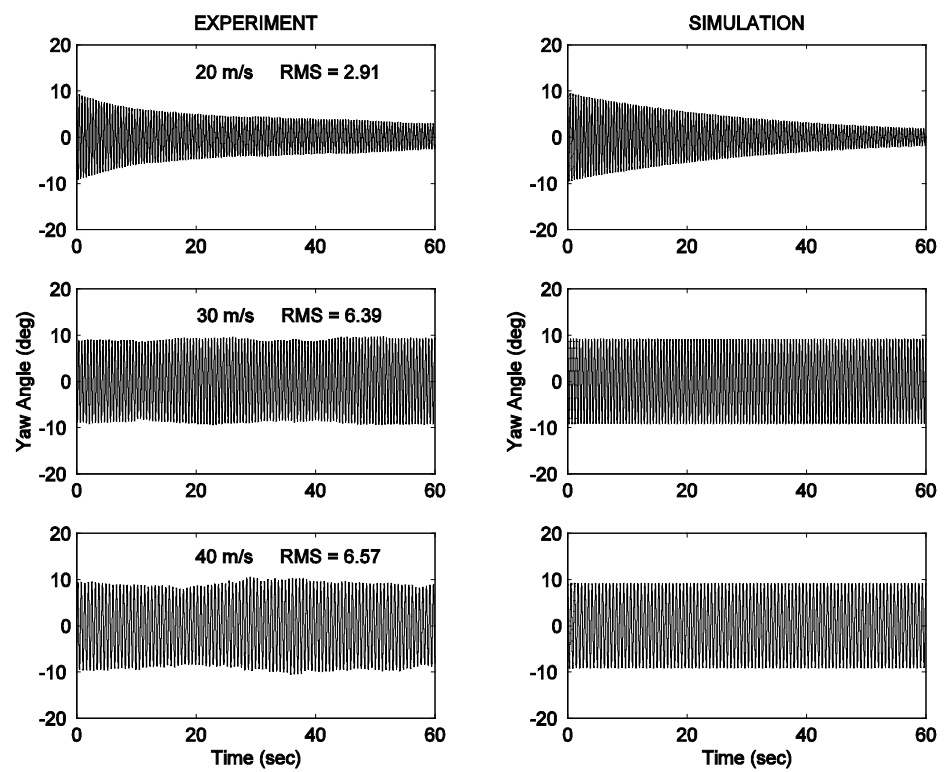

Figure 14 Comparison of measured responses during self sustained oscillation (K6).

However the results from Fig.14 suggest that is not the case and that further analysis is required. In future work it is intended to apply statistical techniques to identify and characterise the unsteady behaviour.

\section{Conclusions}

1. The yaw moment magnification determined from the transient experiment is greater than unity across the reduced frequency range.

2. From quasi-steady simulation and experiment the self-sustained oscillation occurs when the positive aerodynamic damping exceeds the rig mechanical damping.

3. The self-sustained oscillation suggests the existence of unsteady forces due to vortex shedding or self-excited forces that are coupled with the model motion.

4. Although the yaw moment derivative derived using classical techniques appears to become constant at higher speeds this does not account for additional unsteady effects. This is the subject of future study.

\section{Acknowledgements}

The authors would like to thank Mr. K Coulthard, Mr. R Hunter, Mr. P Stinchcombe, Mr. P Reeves and Mr. G Cunningham for their help in the construction of the rig and models.

\section{References}

Bearman P W, Mullarkey S P, 1994, Aerodynamic Forces on Road Vehicles due to Steady Side Winds and Gusts, RAeS, Vehicle Aerodynamics Conference, Loughborough.

Chadwick A, Garry, K P, Howell J, 2000, Transient Aerodynamic Characteristics of Simple Vehicle Shapes by the Measurement of Surface Pressure, Vehicle Aerodynamics SAE SP-1524, 2000-01-0876. 
Garry K P, Cooper K R, 1986, Comparison of Quasi-Static and Dynamic Wind Tunnel Measurements of Simplified Tractor-Trailer Models, Journal of Wind Engineering and Industrial Aerodynamics, No. 22.

Goetz H, 1995, Crosswind Facilities and Procedures, SAE SP-1109, Warrendale.

Hales F D, 1969, Stability Problems of Road Vehicles", 1st Symposium on Road Vehicle Aerodynamics, The City University, London.

Macklin A R, Garry K P, Howell J P, 1996, Comparing Static and Dynamic Testing Techniques for the Crosswind Sensitivity of Road Vehicles, SAE 960674.

Passmore M A, Richardson S, Imam A, 2001, An Experimental Study of Unsteady Vehicle Aerodynamics, IMechE Part D Journal of Automotive Engineering, Volume 215 Number 7.

Russell J B, 1969, Aerodynamic Effects on the Lateral Control and Stability of Motor Vehicles, 1st Symposium on Road Vehicle Aerodynamics, The City University, London.

Ryan A, Dominy, R G, 1998, The Aerodynamic Forces Induced on Passenger Vehicle in Response to a Transient Cross-Wind Gust at a Relative Incidence of $30^{\circ}$, Developments in Vehicle Aerodynamics, SAE SP-1381, 980392. 